\title{
The use of neural network algorithms for modeling injection doses of modern fuel injectors
}

The article presents the possibilities of using artificial intelligence methods to model the injection doses of a modern Common Rail (CR) fuel injector. The presented neural network solution belongs to the experimental models known as black boxes in mechatronics. The backpropagation algorithm and its Levenberg-Marquardt expansion were used for the simulation. The analysis showed that there is a good match between the measurements and the computational model. The proposed solution can be used in the processes of diagnosing not only elements of the injection equipment, but also the internal combustion engine.

Key words: fuel injector, CI engine, Common Rail system, neural network, engine diagnosing

\section{Introduction}

The fuel injector is a device whose task is to distribute and spray fuel in the combustion chamber of the engine. The operating conditions in which it operates are extreme due to the pressures and temperatures inside the engine. The problems related to its deficiencies should be considered in terms of destructive processes coming from the outside (working conditions) and from inside (quality and pollution from the fuel). The output operating parameters of the fuel injector are the injection dose amount and the fuel injection delay. These parameters directly affect the characteristics of the internal combustion engine. As a result of the operation of the vehicle, the operating parameters of the fuel injector change. The process of diagnosis consists in analyzing them and assessing whether they are normal or not. However, standard diagnostics include individual points on the overall operating characteristics of the fuel injector. The research of the authors of the paper has shown that in some cases the analysis of individual points may be insufficient to diagnose a fuel injector. This applies in particular piezo injectors.

In [1], the authors presented a problem with the diagnosis of a vehicle with Continental VDO Siemens piezoelectric injectors installed. The engine ran unevenly at the speed of 1300-1800 rpm. The analysis showed that one of the fuel injectors generated too high injection dose in the pressure range of $40-90 \mathrm{MPa}$ at the control time of $200 \mu \mathrm{s}$. The diagnosis of this fault took a long time because the tested injector was working properly during the standard test. In the paper [2], the authors proposed a method of diagnosing modern fuel injectors by analyzing the temperature of its body [3]. As a result of the leakage, the overflow dose increases, the liquid flow velocity inside the injector increases, which results in an increase in the areas of increased temperature leakage. The research on the diagnosis of CR injectors with the use of overflows has been described in [4]. By modifying the test procedure, it is possible to shorten the diagnosis time of the fuel injectors, achieving its very high accuracy by using artificial intelligence algorithms and implementing it into the test bench. The task of this process is to improve the methods of diagnosing elements of injection equipment by analyzing dosing areas omitted by the standard research process.
In the paper, the authors proposed the use of a neural network to model the injection doses of the selected research object. The proposed computational model can be compared to the experimental black box method [5] used in mechatronics. According to the authors of the above monograph, the input and output parameters of the research object as well as its structure and operation are known in the black box models (experimental), but the internal dimensions are not known. This method can be implemented to model injection doses of modern fuel injectors (Fig. 1).

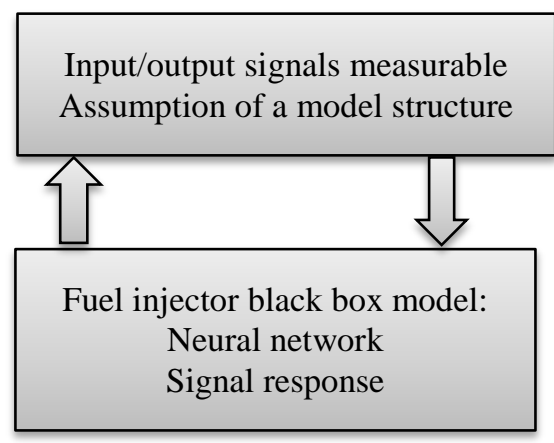

Fig. 1. Black box experimental model [2]

The antagonistic theoretical modeling method in mechatronics is the white box method. To use this method, it is necessary to know the internal dimensions and dependencies in the operation of the tested object. By analyzing the structure and operation of the kinematic associations of modern fuel injectors, it can be concluded that the preparation of the theoretical model called the white box requires precise measurements of the elements of precise pairs, diameters and lengths of the channels in the atomizer and the body. This process is difficult to implement, it is individual for each fuel injector and requires specialized measuring equipment [6].

By analyzing the literature on the considerations of the artificial intelligence method were used to study internal combustion engines. In the work [7], the Bayesian and back propagation algorithms were used to diagnose a compression-ignition engine. The engine was tested at various rotational speeds. The results of the analysis showed that the 
back propagation algorithm simulates the engine operating parameters more accurately than the Bayesian network. By analyzing the results of the experiment, it can be concluded that it is possible to use artificial intelligence methods to supervise the operation of internal combustion engines. The authors of the paper [8] implemented a neural network to predict combustion characteristics for an engine powered by a vegetable fuel. Artificial intelligence prediction was compared with the AVL simulation model. The results of the experiment were similar. To monitor the technical condition of a compression ignition engine, a neural network with the Random Convolutional Neural Network (RCNN) structure was implemented and described in [9]. Based on engine vibration during operation, artificial intelligence assessed its degree of wear. In order to detect early ignitions and knocking as a result, the authors of [10] developed an algorithm implementing artificial intelligence. The task of the neural network is to diagnose the combustion process in the engine during operation on the basis of data collected from the controller. The research presented in the paper [11] describes the diagnostic possibilities with the use of neural networks on the basis of engine noise. Based on the changes in engine noise during operation, the artificial intelligence algorithm assessed its technical condition.

It is possible to use artificial intelligence when diagnosing modern fuel injectors and assessing their output parameters. The authors of the paper developed an algorithm that models the size of injection doses using mechatronics methods on the basis of black box modeling.

\section{Presentation of the selected algorithm}

The process of learning neurons consists in calculating the sum of the values of the input products and the corresponding weights. The obtained value is subjected to the action of an appropriately defined activation function to obtain the output neuron. Having reference characteristics, it is possible to define an error at the output of the neuron. A similar method is used for determining the errors for the last layer in the case of multilayer networks. The problem is defining the error value for hidden layers, because without a pattern, the algorithm is not able to determine the size of the neurons for these areas. The error back propagation method should be used to solve this problem. In order to derive this algorithm, the error measure should be defined, which is the function $\mathrm{Q}(\mathrm{w})$. In this function, the variables are all weights of the multilayer neural network. The training of the network consists in finding the minimum of the function $\mathrm{Q}$ with respect to the vector $\mathrm{w}$. Then the function should be expanded into the Taylor series in the closest vicinity of the known current solution $\mathrm{w}$ along the $\mathrm{p}$ direction, relation (1) [2].

$\mathrm{Q}(\mathrm{w}+\mathrm{p})=\mathrm{Q}(\mathrm{w})+|\mathrm{g}(\mathrm{w})|^{\mathrm{T}} \mathrm{p}+0,5 \mathrm{p}^{\mathrm{T}} \mathrm{H}(\mathrm{w}) \mathrm{p}+\cdots \ldots$,

where $g(w)$ is the gradient vector and $\mathrm{H}(\mathrm{w})$ is a matrix of second derivatives.

In general, the algorithm of the back propagation error method is written as follows (2)-(5) [9]:

$\mathrm{y}_{\mathrm{i}}^{(\mathrm{k})}(\mathrm{t})=\mathrm{f}\left(\mathrm{s}_{\mathrm{i}}^{(\mathrm{k})}(\mathrm{t})\right), \mathrm{s}_{\mathrm{i}}^{(\mathrm{k})}(\mathrm{t})=\sum_{\mathrm{j}=0}^{\mathrm{N}_{\mathrm{k}-1}} \mathrm{w}_{\mathrm{ij}}^{(\mathrm{k})}(\mathrm{t}) \mathrm{x}_{\mathrm{j}}^{(\mathrm{k})}(\mathrm{t})$

$$
\begin{aligned}
& \mathrm{Q}_{\mathrm{i}}^{(\mathrm{k})}(\mathrm{t})=\left\{\begin{array}{c}
\mathrm{d}_{\mathrm{i}}^{(\mathrm{L})}(\mathrm{t})-\mathrm{y}_{\mathrm{i}}^{(\mathrm{L})}(\mathrm{t}) \text { dlak }=\mathrm{L} \\
\sum_{\mathrm{m}=1}^{\mathrm{N}_{\mathrm{k}+1}} \delta_{\mathrm{m}}^{(\mathrm{k}+1)}(\mathrm{t}) \mathrm{w}_{\mathrm{mi}}^{(\mathrm{k}+1)(\mathrm{t})} \text { dlak }=1, \ldots, \mathrm{L}-1
\end{array}\right. \\
& \delta_{\mathrm{i}}^{(\mathrm{k})}(\mathrm{t})=\varepsilon_{\mathrm{i}}^{(\mathrm{k})}(\mathrm{t}) \mathrm{f}^{\mathrm{l}}\left(\mathrm{s}_{\mathrm{i}}^{(\mathrm{k})}(\mathrm{t})\right) \\
& \mathrm{w}_{\mathrm{ij}}^{(\mathrm{k})}(\mathrm{t}+1)=\mathrm{w}_{\mathrm{ij}}^{(\mathrm{k})}(\mathrm{t})+2 \eta \delta_{\mathrm{i}}^{(\mathrm{k})}(\mathrm{t}) \mathrm{x}_{\mathrm{j}}^{(\mathrm{k})}(\mathrm{t})
\end{aligned}
$$

The operation of the algorithm starts at the moment of providing the training pattern to the network input. First, it is processed by the neurons of the first layer which determine the output signal. The signals obtained in this way are inputs for the neurons of the next layer. This cycle continues until the last layer. Having known the output signal of the last layer and the reference signal from the training sequence, it is possible to calculate the error at the network output from the dependence (2). Then, the weights of the last layer neurons are modified using the dependencies (2)-(4). The output error is propagated backwards according to the connections of neurons between the layers and taking into account their dependency activation function (2), (3). The second algorithm on the basis of which the neural network presented in the article was generated is the Levenberg-Marquardt algorithm. It uses the expansion of the function $\mathrm{Q}(\mathrm{w})$ expressed by the formula (1) to the third component [5].

\section{Presentation of the results of measurements and simulations}

Laboratory tests were performed using a test bench for testing injection pumps and STPiW3 fuel injectors. The research object was an electromagnetic fuel injector from Bosch, serial number 0445110083, generation 1.0.

The standard operating characteristics of the tested fuel injector were made on the test bench (Fig. 2).

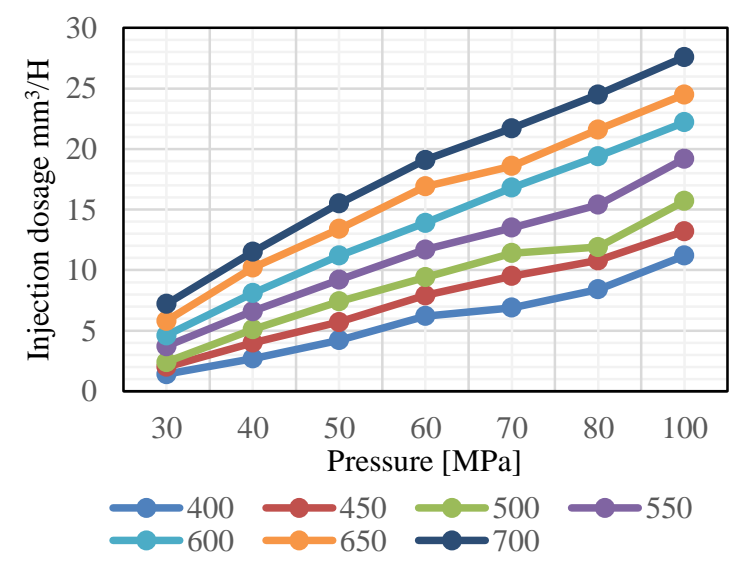

Fig. 2. Standard operating characteristics of the tested fuel injector - parameters measured on the test bench

Then, the second operating characteristic was performed, but with the injector control times changed (Fig. 3).

Based on the parameters from the standard operating characteristics of the fuel injector (Fig. 2), the neural network modeled the injection doses for the characteristic with changed control times (Fig. 4). 


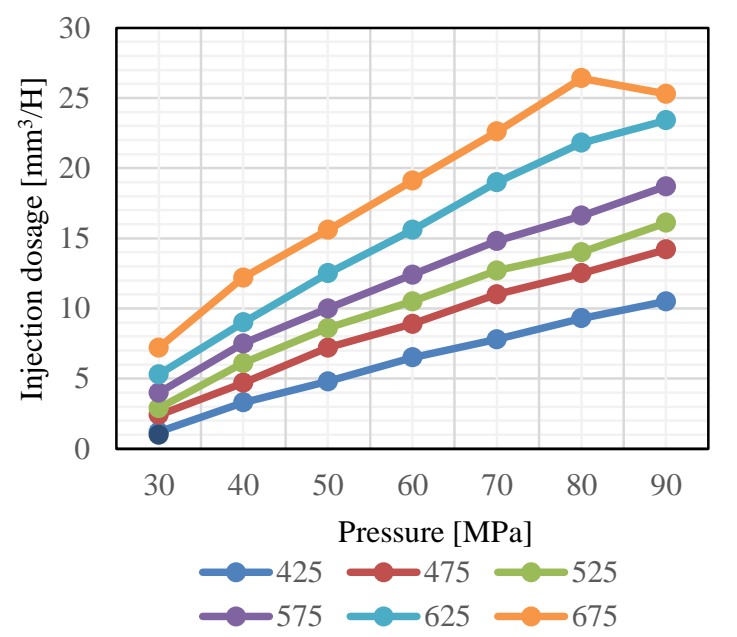

Fig. 3. Performance characteristics of the tested fuel injector - parameters measured on the test bench

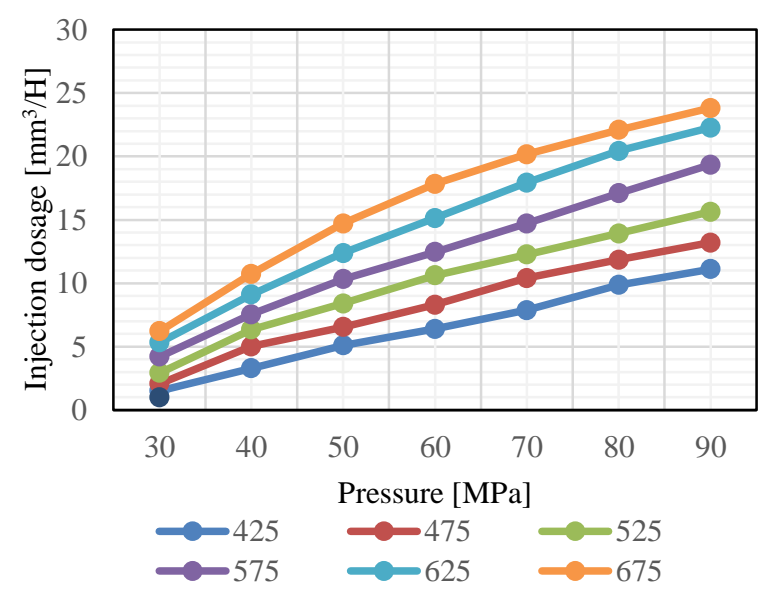

Fig. 4. Operating characteristics of the tested fuel injector-parameters modeled by the neural network

Figures 5, 6 and 7 show the parameters for selected fuel injector control times. In Figures 5 and 6, the fit of the model is very high, while in 7 , slight differences occur for pressures of 70 and $80 \mathrm{MPa}$.

Injection time $475 \mu$ s neural network

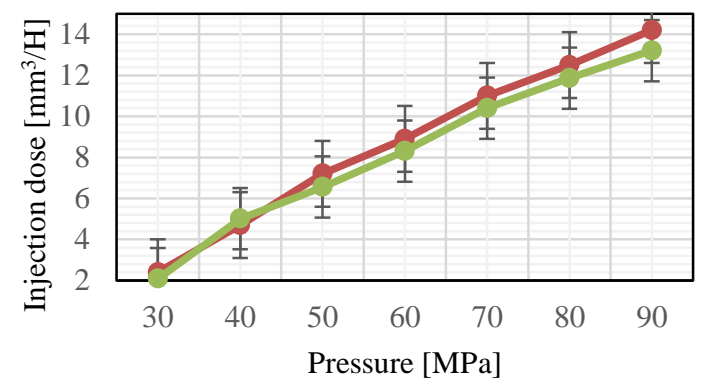

$\longrightarrow$ Measured values $\longrightarrow$ Calculated values

Fig. 5. Comparison of measured and calculated parameters for the injector control time of $475 \mu \mathrm{s}$
Injection time $525 \mu$ s neural network

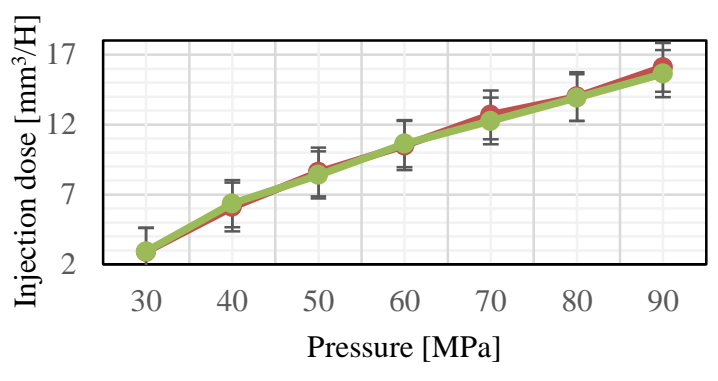

-Measured values $\longrightarrow$ Calculated values

Fig. 6. Comparison of measured and calculated parameters for the injector control time of $525 \mu \mathrm{s}$

\section{Injection time $675 \mu$ s neural network}

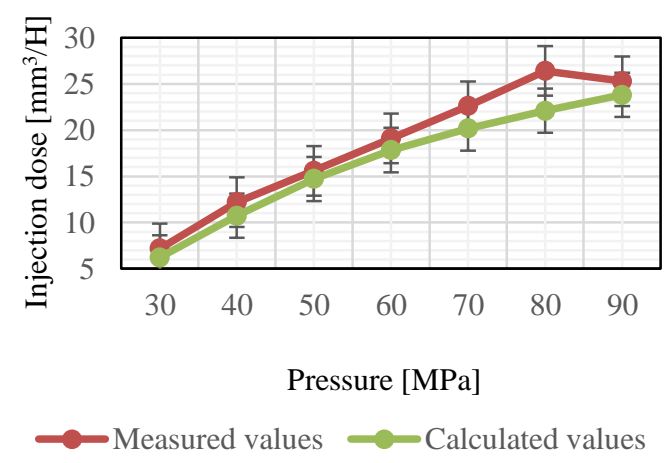

Fig. 7. Comparison of measured and calculated parameters for the injector control time of $675 \mu \mathrm{s}$

\section{Discussion of research results}

The model of the neural network proposed by the authors consists of two inputs (pressure in the system and fuel injection time) and one output: injection dose size. This network consists of three hidden layers, of which the first and the third layer have five neurons each, and the second layer contains four neurons. The last layer is the output layer, which consists of one neuron. The number of layers depends on the complexity of the model (variables). A single neuron divides the plane into two parts. Two layers can represent simplexes, i.e. convex areas limited by hyperplanes. The three-layer network is able to define any area, therefore a network model with three hidden layers was chosen. The selection of the number of neurons in a given layer was selected according to Kolmogorov theorem: the number of neurons in a layer should be $2 n+1$ where $\mathrm{n}$ is the number of inputs. So our network should have 5 neurons in each hidden network. However, during the tests it was noticed that the combination of 5-5-5 does not reflect the model perfectly. Then, the algorithm of back propagation of errors was selected, which is to select appropriate weights for individual neurons during their training, and its modification, the Levenberg-Marquardt algorithm. A neural network model with five neurons in each hidden layer is not an optimal solution. For the purposes of the research, an algorithm aimed at creating a neural net- 
work and training it was modified, which consisted in finding the best structure, i.e. selecting such a number of neurons in each of the three layers that the mean absolute error was as small as possible, determined by the relationship (6):

$$
\mathrm{sbb}=\left(\sum(|\mathrm{Xi}|-|\mathrm{Yi}|)\right) / \mathrm{n}
$$

where: $\mathrm{sbb}$ - mean absolute error, $\mathrm{Xi}$ - injector dose obtained during measurements on the test bench, $\mathrm{Yi}$ - injector dose determined from the test bench, $\mathrm{n}$ - number of doses tested.

With three layers of five neurons each, the authors created an algorithm that checked 125 possible combinations, assuming the number of neurons in each layer from one to five. In addition, each network structure was trained five times and their mean absolute error was calculated to reduce the impact of the randomness of selecting weights at the beginning of learning a given structure. Our tests and analyzes show that building a neural network with a structure of 5-5-5 is suboptimal and burdened with high risk, and that the network used will be inaccurate.

Table 1. Results of the mean absolute error of the neural network 5-5-5

\begin{tabular}{|c|c|c|c|c|}
\hline & 1 & 2 & 3 & 4 \\
\hline 621 & 0.9180 & 5 & 5 & 5 \\
\hline 622 & 1.622 & 5 & 5 & 5 \\
\hline 623 & 2.5320 & 5 & 5 & 5 \\
\hline 624 & 0.8839 & 5 & 5 & 5 \\
\hline 625 & 0.7564 & 5 & 5 & 5 \\
\hline
\end{tabular}

Table 1 shows the values of the mean absolute errors for a particular network training attempt. In the best case, the error was 0.07564 and in the worst one it was four times higher. This structure is not very stable. Finally, the mean of all errors is 1.2505 . It is far too high. Our criterion was that the error should not exceed 1 . Table 2 shows the structure of the network 5-4-5 chosen as the most optimal.

Table 2. Results of the mean absolute error of the neural network 5-4-5

\begin{tabular}{|c|c|c|c|c|}
\hline & 1 & 2 & 3 & 4 \\
\hline 596 & 0.7973 & 5 & 4 & 5 \\
\hline 597 & 0.8165 & 5 & 4 & 5 \\
\hline 598 & 0.7605 & 5 & 4 & 5 \\
\hline 599 & 0.9139 & 5 & 4 & 5 \\
\hline 600 & 0.9960 & 5 & 4 & 5 \\
\hline
\end{tabular}

The smallest error is 0.7605 and the largest one is 0.9960 , the maximum difference is 0.2355 . The error values are very close with each learning attempt. Additionally, their value does not exceed 1 . Out of all 125 combinations this network structure, i.e. 5-4-5, is the most optimal for our tests.

The analysis of the research carried out on the various combinations presents:

- the best structures of a neural network, in which the error is as small as possible, are obtained in networks in which at least one hidden layer has 5 neurons,

- the structure 5-5-5 is not very stable because during each learning the mean absolute error may differ several times from the previous learning,
- Kolmogorov's statement is not accurate and may serve as a guideline for the selection of the shape of the neural network.

After selecting the appropriate structure of the neural network, the process of its training began in order to achieve the lowest possible error. Structural science studies have shown that the lowest absolute error was achieved at the level of 0.6682 .

Table 3 shows the error values between the output parameters measured and calculated for each input data.

Table 3. The size of the error between the parameters measured on the test bench and the model of the neural network structure

\begin{tabular}{|c|c|c|c|c|c|c|c|}
\hline & 30 & 40 & 50 & 60 & 70 & 80 & 90 \\
\hline 425 & -0.32 & 0.01 & -0.30 & 0.09 & -0.08 & -0.56 & -0.61 \\
\hline 475 & 0.32 & -0.31 & 0.65 & 0.59 & 0.60 & 0.64 & 0.99 \\
\hline 525 & -0.02 & -0.26 & 0.20 & -0.13 & 0.44 & 0.07 & 0.47 \\
\hline 575 & -0.21 & -0.05 & -0.33 & -0.06 & 0.09 & -0.49 & -0.64 \\
\hline 625 & -0.03 & -0.10 & 0.12 & 0.45 & 1.06 & 1.37 & 1.12 \\
\hline 675 & 0.97 & 1.47 & 0.88 & 1.26 & 2.42 & 4.30 & 1.49 \\
\hline 725 & -0.15 & 0.53 & 0.67 & 1.63 & 1.24 & 0.68 & 1.27 \\
\hline
\end{tabular}

Analyzing the above results, the average error is 0.48 $\mathrm{mm}^{3} / \mathrm{H}$, the maximum error is $4.30 \mathrm{~mm}^{3} / \mathrm{H}$ and the minimum one is $-0.64 \mathrm{~mm}^{3} / \mathrm{H}$.

There are known computational methods for analyzing the amount of injection doses using polynomials. The disadvantage of these methods is that the input quantities are limited (only two data can be used). When using artificial intelligence methods, any number of inputs can be used, which is very important in diagnostic processes.

\section{Summary}

The analysis of simulation tests showed that it is possible to use artificial intelligence (AI) methods to model the size of injection doses of modern fuel injectors. The implementation of the artificial intelligence algorithm is characterized by a high fit, therefore the results of mathematical modeling are similar to the experimental ones. The aim of the simulation was to investigate whether it is possible to model the output parameters of a modern fuel injector without knowing its internal dimensions on the basis of modeling a standard operating characteristic. The Neural Network add-on of the Matlab environment was used to make the model.

Further work carried out by the authors of the paper is aimed at improving the artificial intelligence algorithm by introducing additional input variables. The modification will consist in indicating to the algorithm how individual elements of the fuel injector affect the size of injection doses in individual areas of its operating characteristics. Additionally, the algorithm will be implemented in the test bench controller. The proposed algorithm can be used in the diagnostics of other engine components and the analysis of its current parameters during operation. The analytical methods proposed by the authors represent an innovative approach to prediction of injection doses of a selected fuel injector. 


\section{Nomenclature}

CI compression ignition

CR common rail

sbb mean absolute error

$\mathrm{Xi} \quad$ injector dose obtained from measurements on the test bench
$\mathrm{Yi} \quad$ injector dose modeled from the test bench

$\mathrm{N}$ number of samples

NN Neural Network

\section{Bibliography}

[1] OSIPOWICZ, T., ABRAMEK, K.F. Diagnosing methods common rail fuel injectors. Combustion Engines. 2017, 168(1), 56-61. https://doi.org/10.19206/CE-2017-109

[2] OSIPOWICZ, T., ABRAMEK, K.F. The analysis of temperature disintegration on the body of fuel injector during research on test bench. Combustion Engines. 2017, 168(1), 172-177. https://doi.org/10.19206/CE-2017-128

[3] ELIASZ, J., OSIPOWICZ, T., ABRAMEK, K.F., MOZGA, Ł. Model issues regarding modification of fuel injector components to improve the injection parameters of a modern compression ignition engine powered by biofuel. Applied Sciences. 2019, 9(24), 5479. https://doi.org/10.3390/app9245479

[4] KNEFEL, T. Ocena techniczna wtryskiwaczy Common Rail na podstawie doświadczalnych badań przelewów. Eksploatacja i Niezawodnosc - Maintenance and Reliability. 2012, 14(1), 42-53.

[5] RUTKOWSKI, L. Metody i techniki sztucznej inteligencji. Wydawnictwo Naukowe PWN. Warszawa 2012.

[6] MARCIC, S., MARCIC, M., PRAUNSEIS Z. Mathematical model for the injector of a common rail fuel - injection system. Engineering. 2015, 7(6).

https://doi.org/10.4236/eng.2015.76027

[7] CAI, B., SUN, X., WANG, J. et al. Fault detection and diagnostic method of diesel engine by combining rule -

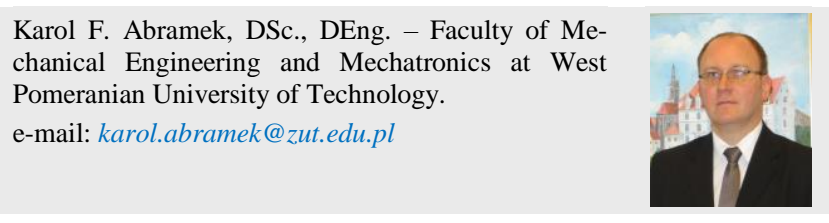

Tomasz Osipowicz, DEng. - Faculty of Mechanical Engineering and Mechatronics at West Pomeranian University of Technology.

e-mail: tosipowicz@zut.edu.pl based algorithm and BNs/BPNNs. Journal of Manufacturing Systems. 2020, 57(7), 148-157.

https://doi.org/10.1016/j.jmsy.2020.09.001

[8] KARAMI, R., RASUL, M.G., MASUD, M. et al. Experimental and computational analysis of combustion characteristics of a diesel engine fueled with diesel-tomato seed oil biodiesel blends. Fuel. 2021, 285, 119243. https://doi.org/10.1016/j.fuel.2020.119243

[9] WANG, R., CHEN, H., GUAN, C. Random convolutional neural network structure: An intelligent health monitoring scheme for diesel engines. Measurement. 2021, 171, 108786. https://doi.org/10.1016/j.measurement.2020.108786

[10] KUZHAGAliYeVA, N., THABET, A., SINGH, E. et al. Using deep neural networks to diagnose engine preignition. Proceedings of the Combustion Institute. 2020, 38(4), 5915-5922.

https://doi.org/10.1016/j.proci.2020.10.001

[11] WANG, Y., LIU, N.N., GUO, H. et al. An engine-faultdiagnosis system based on sound intensity analysis and wavelet packet pre-processing neural network. Engineering Applications of Artificial Intelligence. 2020, 94, 103765.

https://doi.org/10.1016/j.engappai.2020.103765

Łukasz Mozga, MSc. - Faculty of Mechanical Engineering and Mechatronics at West Pomeranian University of Technology.

e-mail: lukasz.mozga@zut.edu.pl 